[Original]

\title{
Eplerenone Improves Vascular Endothelial Function in Patients with Primary Aldosteronism: A Pilot Study
}

\author{
Akira Kurozumi ${ }^{*}$ Yosuke OKada and Yoshiya Tanaka \\ First Department of Internal Medicine, School of Medicine, University of Occupational and Environmental Health, \\ Japan. Yahatanishi-ku, Kitakyushu 807-8555, Japan
}

\begin{abstract}
Primary aldosteronism (PA) is associated with a high risk of cardiovascular complications. Large-scale clinical studies have demonstrated that mineralocorticoid receptor antagonists (MRA) exhibit organ-protective effects and improve the prognosis of patients with heart failure and myocardial infarction, and daily clinical practice suggests that MRA seem to improve vascular endothelial dysfunction. In this pilot study, we treated 10 PA patients with eplerenone for 3 months. We used Endo-PAT to evaluate the effects of MRA on vascular endothelial function and analyzed the data for correlative factors. The primary outcome measure, the reactive hyperemia index (RHI), was 1.71 before therapy and increased significantly to 2.21 . Univariate analysis showed a significant correlation between the rate of change in RHI and that in plasma renin activity (PRA). Since plasma aldosterone concentration increases during MRA therapy, PRA may be the best marker for selecting the most appropriate dose of MRA. PRA can potentially be used for adjusting the dose of MRA, in addition to adjusting blood pressure and serum potassium level.
\end{abstract}

Keywords : primary aldosteronism, endothelial dysfunction, reactive hyperemia index, eplerenone.

(Received May 10, 2021, accepted June 14, 2021)

\section{Introduction}

Primary aldosteronism (PA) is a typical form of secondary hypertension and is the underlying etiology of hypertension in approximately $4-19 \%$ of cases [13]. PA is reported to be associated with a high risk of cerebrovascular and cardiovascular diseases compared to essential hypertension (EH) in patients of similar age and blood pressure $[4,5]$. According to the 2019 Japanese Society of Hypertension guidelines for the management of hypertension, drug therapy with mineralocorticoid receptor (MR) antagonists (MRA) as the first choice of treatment should, in principle, be administered continuously throughout life to patients who have bilateral PA, do not wish to undergo surgery, are inoperable, or do not wish to take any tests after screening [6].

MR is expressed in various organs in addition to the distal renal tubules. The high incidence of cardiovascular complications due to aldosterone may be directly related to inflammation or increased oxidative stress in the vascular endothelium [7]. Since aldosterone itself has a damaging effect on the cardiovascular system, large-scale clinical studies have demonstrated that MRA exhibits an organ-protective effect and improves the prognosis of patients with heart failure or myocardial infarction $[8,9]$. Few studies, however, have reported that MRA improves vascular endothelial func-

*Corresponding Author: Akira Kurozumi, MD, PhD, First Department of Internal Medicine, School of Medicine, University of Occupational and Environmental Health, Japan. 1-1 Iseigaoka, Yahatanishi-ku, Kitakyushu 807-8555, Japan, Tel: +81-93-603-1611, Fax: +81-93-691-9334, E-mail: akira@med.uoeh-u.ac.jp 
tion, i.e., the early stage of arteriosclerosis, in clinical practice. Endo-PAT (Endo-PAT2000, Itamar Medical, Caesarea, Israel) using peripheral arterial tonometry (PAT) has been used as a noninvasive method for the assessment of vascular endothelial function [10], and the usefulness of reactive hyperemia index (RHI) in predicting vascular diseases has been reported [11] We previously assessed the relation of endothelial function in clinical studies [12-17], but clinical trials using RHI in PA are limited compared to those using flow-mediated dilation (FMD). In this study we treated 10 patients with PA by using eplerenone for 3 months, and used Endo-PAT to evaluate the effects of MRA on the status of vascular endothelial function and explored the factors that correlate with the improvement of this function.

\section{Materials and Methods}

\section{Participants}

The study participants were 10 patients with PA, who visited the Outpatient Clinics of the Department of Endocrinology, Metabolism and Diabetes, University of Occupational and Environmental Health, between October 2017 and December 2018. They were not using drugs that affect the aldosterone-renin ratio (ARR), such as angiotensin converting enzyme (ACE) inhibitors or angiotensin II receptor blockers (ARB) at the time of endocrinological examination, but they were being treated with calcium channel blockers and/ or $\alpha$-blockers. PA was suspected based on screening data of $A R R \geq 200$. Each participant underwent a captopril load test, a physiological saline load test, and a furosemide standing test, and the diagnosis of PA was confirmed when two or more of the above three tests, which are listed in the Japanese Endocrine SocietyPA Diagnostic and Treatment Guideline 2009, proved positive. We excluded patients with diabetes and cardiac arrhythmia on electrocardiogram. For localized diagnosis, adrenal computed tomography (CT) was performed in all 10 patients. None had adrenal tumors on either side. Selective adrenal venous sampling (AVS) was performed in 6 patients and confirmed the presence of bilateral PA. This study was approved by the Ethics Committee of the University of Occupational and Environmental Health, Japan (H27-186).

\section{Study Design}

This retrospective study included 10 patients with PA who were treated with $50 \mathrm{mg} /$ day eplerenone. Each patient underwent the Endo-PAT before and 3 months after treatment with eplerenone to measure their RHI. No changes were made to other medications except for anti-hypertensive drugs (calcium channel blockers were reduced or discontinued in 4 cases, and $\alpha$-blocker was discontinued in 1 case). Blood pressure was evaluated by blood pressure in the examination room, not by home blood pressure. The primary endpoint was changes in RHI after treatment with eplerenone, and the secondary endpoint was the correlation between changes in RHI and changes in plasma aldosterone concentration (PAC), plasma renin activity (PRA) and ARR.

Assessment of endothelial function with Endo-PAT

We assessed vascular function in 10 patients by Endo-PAT. The method used for digital assessment of vascular function using Endo-PAT has been described elsewhere [18].

\section{Statistical analysis}

Data were expressed as mean $\pm \mathrm{SD}$. The Wilcoxon test was used for comparison of pre-treatment with the 3 months of treatment (Figure 1, Table 1). The Spearman correlation test was used for analysis of the data listed in Table 2. The level of significance was set at $P<0.05$. All statistical analyses were conducted using the Statistical Package for Social Association version 21.0 (SPSS Inc., Chicago, IL).

\section{Results}

The baseline characteristics of the patients are shown in Table 3. The study participants were 10 patients with PA ( 3 males; 7 females). The mean age of the participants was 60.4 years. The participants were mildly obese, with mean body mass index of $25.5 \mathrm{~kg} / \mathrm{m}^{2}$. Renal function was normal, with eGFR of $77.3 \mathrm{ml}$ $\min / 1.73 \mathrm{~m}^{2}$. Laboratory tests showed almost normal serum potassium (only 1 patient had hypokalemia).

As shown in Figure 1, the primary outcome measure, the RHI value was $1.71 \pm 0.41$ before therapy, but increased significantly to $2.21 \pm 0.62$ at 3 months after 


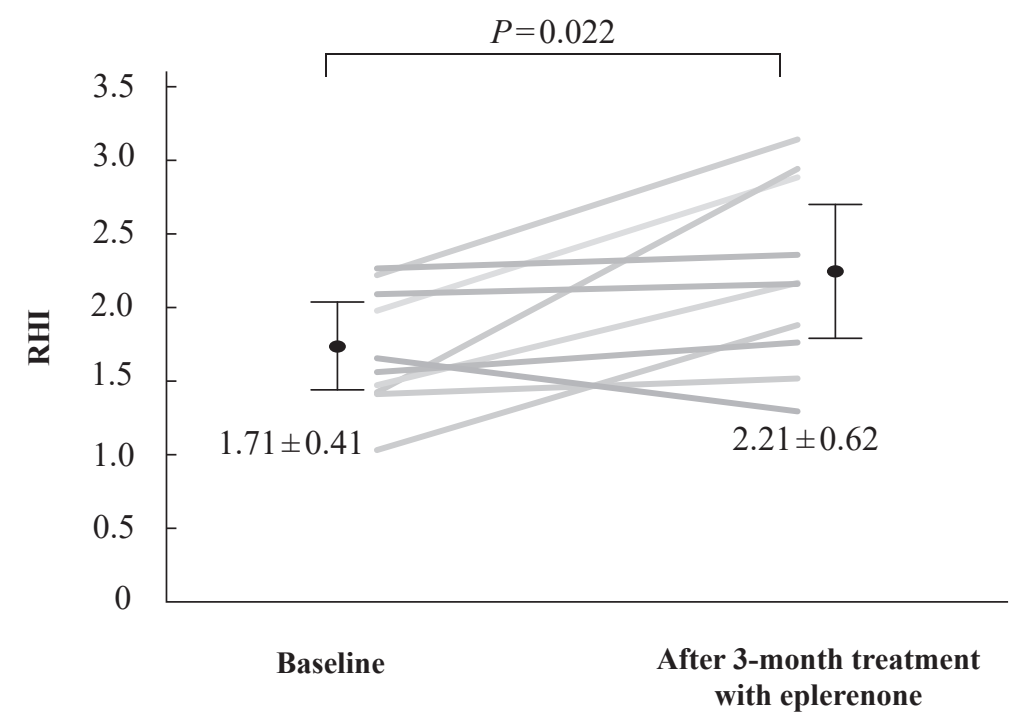

Figure 1. Vascular endothelial function before and after 3-month treatment with eplerenone. RHI: reactive hyperemia index.

Table 1. Effects of 3-month treatment with eplerenone on various parameters

\begin{tabular}{lccc}
\hline & Before therapy & After therapy & $P$ value \\
\hline Clinical parameters & & & \\
SBP $(\mathrm{mmHg})$ & $149.7 \pm 19.8$ & $136.1 \pm 19.0$ & 0.221 \\
DBP $(\mathrm{mmHg})$ & $93.4 \pm 12.4$ & $81.8 \pm 13.8$ & 0.083 \\
Biochemical parameters & & & \\
PAC $(\mathrm{pg} / \mathrm{m} l)$ & $125.7 \pm 54.7$ & $192.0 \pm 82.4$ & 0.037 \\
PRA $(\mathrm{ng} / \mathrm{m} / / \mathrm{hr})$ & $0.32 \pm 0.20$ & $0.94 \pm 0.51$ & 0.011 \\
ARR & $452 \pm 245$ & $326 \pm 345$ & 0.203 \\
K $(\mathrm{mEq} / l)$ & $3.9 \pm 0.3$ & $4.3 \pm 0.5$ & 0.028 \\
\hline
\end{tabular}

Data are mean \pm SD. $P$ values for comparison of before and after therapy, by Wilcoxon test. SBP: systolic blood pressure, DBP: diastolic blood pressure, PAC: plasma aldosterone concentration, PRA: plasma renin activity, ARR: aldosterone-renin ratio.
Table 2. Correlation between $\Delta$ change in RHI and $\Delta$ change in various parameters

\begin{tabular}{lcc}
\hline Variable & \multicolumn{2}{c}{ Univariate Analysis } \\
\cline { 2 - 3 } & $\mathrm{r}$ & $P$ value \\
\hline SBP & -0.479 & 0.162 \\
DBP & -0.261 & 0.467 \\
K & 0.164 & 0.651 \\
PAC & -0.370 & 0.293 \\
PRA & 0.939 & $<0.001$ \\
ARR & -0.915 & $<0.001$ \\
\hline
\end{tabular}

Data are $P$ value by Spearman rank correlation. RHI: reactive hyperemia index, SBP: systolic blood pressure, DBP: diastolic blood pressure, PAC: plasma aldosterone concentration, PRA: plasma renin activity, ARR: aldosterone-renin ratio. treatment with eplerenone $(P=0.022)$. There were no significant changes in blood pressure and ARR (Table 1), but PAC, PRA and serum potassium significantly increased after the 3-month treatment. With regard to the secondary outcome measures, univariate analysis of factors affecting the $\Delta$ change in RHI identified significant correlations with the $\Delta$ change in PRA and ARR (Table 2).

\section{Discussion}

The main findings of the present study based on the use of Endo-PAT were that 3-month continuous treatment with MRA improved vascular endothelial function in patients with PA, and that the changes in this function correlated with changes in PRA.

The American Endocrine Society revised the clinical practice guidelines for the diagnosis and treatment of PA in 2016, for the first time in 8 years [19]. The revised guidelines recommended treatment with MRA for patients with positive results in the first screening test who do not wish to or cannot be further tested. Treatment with MRA is also recommended for patients with suspected PA, thus confirming the importance of 
Table 3. Baseline characteristics of patients

\begin{tabular}{|c|c|}
\hline Valiable & mean \pm SD or number \\
\hline sex (male:female) & $3: 7$ \\
\hline age (years) & $60.4 \pm 12.8(41-76)$ \\
\hline body mass index $\left(\mathrm{kg} / \mathrm{m}^{2}\right)$ & $25.5 \pm 3.6(21.8-33.1)$ \\
\hline Systolic blood pressure (mmHg) & $146.2 \pm 17.4(112-168)$ \\
\hline Diastolic blood pressure (mmHg) & $90.5 \pm 8.6(76-107)$ \\
\hline LDL-C (mg/d $l)$ & $125.3 \pm 29.6(77-182)$ \\
\hline HDL-C (mg/d $l)$ & $67.5 \pm 18.5(38-91)$ \\
\hline Triglyceride $(\mathrm{mg} / \mathrm{d} l)$ & $115 \pm 70(42-282)$ \\
\hline Aspartate aminotransferase (IU/l) & $23.8 \pm 9.1(13-41)$ \\
\hline Alanine aminotransferase (IU/l) & $23.1 \pm 17.2(8-67)$ \\
\hline$\gamma$-GTP $(\mathrm{IU} / l)$ & $34.1 \pm 18.8(15-80)$ \\
\hline Cre $(\mathrm{mg} / \mathrm{d} l)$ & $0.70 \pm 0.22(0.44-1.15)$ \\
\hline eGFR $\left(\mathrm{ml} / \mathrm{min} / 1.73 \mathrm{~m}^{2}\right)$ & $77.3 \pm 16.7(57.4-106.2)$ \\
\hline $\mathrm{FPG}(\mathrm{mg} / \mathrm{d} l)$ & $95.7 \pm 7.1(90-109)$ \\
\hline HbA1c $(\%)$ & $5.6 \pm 0.4(4.9-6.1)$ \\
\hline $\mathrm{K}(\mathrm{mEq} / \mathrm{l})$ & $3.9 \pm 0.3(3.2-4.2)$ \\
\hline $\mathrm{PAC}(\mathrm{pg} / \mathrm{m} l)$ & $136 \pm 54(53-213.0)$ \\
\hline PRA (ng/ml/hr) & $0.33 \pm 0.22(0.2-0.9)$ \\
\hline ARR & $480.8 \pm 259.8(230.0-1065.0)$ \\
\hline $24 \mathrm{hr}$ urine-aldosterone $(\mu \mathrm{g} /$ day $)(\mathrm{n}=8)$ & $9.1 \pm 3.1(4.3-14.3)$ \\
\hline Smoking (n) & 3 \\
\hline Alcohol (n) & 6 \\
\hline Statin (n) & 1 \\
\hline CCB (n) & 9 \\
\hline$\alpha$-blocker (n) & 1 \\
\hline $\mathrm{ARB}(\mathrm{n})$ & 0 \\
\hline
\end{tabular}

Data are mean \pm SD or number (range: minimum-maximum). LDL: low-density lipoprotein, HDL: high-density lipoprotein, $\gamma$-GTP: gamma-glutamyl transpeptidase, Cre: Creatinine, eGFR: estimated glomerular filtration rate, FPG: fasting plasma glucose, HbA1c: hemoglobin A1c, PAC: plasma aldosterone concentration, PRA: plasma renin activity, ARR: aldosterone-renin ratio, CCB: calcium channel blocker, ARB: angiotensin receptor blocker.

medical treatment. Eplerenone, a second-generation MRA with enhanced selectivity for MR, became available in Japan in 2007. It is highly selective for MR and has only a few adverse endocrine reactions, such as gynecomastia, which is observed in patients treated with spironolactone.

Previous studies using FMD reported that PA was associated with poorer vascular endothelial function compared with EH [20-22], and that the percentage of FMD (\%FMD) correlated negatively with PAC, ARR $[19,20]$, and 24-hour urinary aldosterone excre- tion $[21,22]$. It was also reported that MRA therapy and adrenalectomy improved \%FMD in patients with PA [20-22]. In vitro, eplerenone improved endothelial function through the enhancement of the expression of the endothelial nitric oxide (NO) synthase gene [23, 24] suggesting that eplerenone directly increases $\mathrm{NO}$ production in hypertension by activation of endothelial NO synthase. Inhibition of the aldosterone/mineralocorticoid receptor may also contribute to a decrease in oxidative stress, resulting in inhibition of NO inactivation [25]. In vivo, spironolactone improved \%FMD 
and endothelium-dependent vasodilation induced by acetylcholine in patients with hyperaldosteronism and in patients with heart failure [26, 27]. These reports did not examine the factors that contribute to the improvement in \%FMD in patients with PA [20-22]. Although our study was retrospective in nature, it is the first to report on the factors that correlate with MRArelated improvement in vascular endothelial function.

Hundemer et al reported that 602 patients with PA showed 1.91 times higher incidence of cardiovascular events, 1.34 times higher mortality, 1.26 times higher incidence of diabetes mellitus, and 1.93 times higher incidence of atrial fibrillation than age-matched patients with EH [28]. They also reported that treatment with MRA reduced the incidence of cardiovascular events in PA patients with high PRA $(\geq 1.0 \mathrm{ng} /$ $\mathrm{m} / / \mathrm{hr}$ ) to levels comparable to those in EH. In other words, since MRA therapy increases PAC, PRA may be the best marker for selecting the appropriate dose of MRA, as confirmed by our results. The adjustment of the dose of MRA is generally based on serum potassium levels. Based on the results of our study and those of Hundemer et al, PRA can potentially be used for adjusting the dose of MRA, in addition to blood pressure and serum potassium level.

The limitations of the present study are: (1) the small sample size of 10 patients, (2) the retrospective study design, and (3) the lack of a control against eplerenone, such as a placebo. We plan to conduct a prospective study in the near future that will include a control group and a larger sample size.

\section{Conflict of Interest}

The authors declare no conflict of interest.

\section{References}

1. Monticone S, Burrello J, Tizzani D et al (2017): Prevalence and Clinical Manifestations of Primary Aldosteronism Encountered in Primary Care Practice. J Am Coll Cardiol 69 (14): 1811-1820

2. Baudrand R, Guarda FJ, Torrey J, Williams G \& Vaidya A (2016): Dietary Sodium Restriction Increases the Risk of Misinterpreting Mild Cases of Primary Aldosteronism. J Clin Endocrinol Metab 101 (11): 3989-
3996

3. Piaditis G, Markou A, Papanastasiou L, Androulakis, II \& Kaltsas G (2015): Progress in aldosteronism: a review of the prevalence of primary aldosteronism in pre-hypertension and hypertension. Eur J Endocrinol 172 (5): R191-203

4. Savard S, Amar L, Plouin PF \& Steichen O (2013): Cardiovascular complications associated with primary aldosteronism: a controlled cross-sectional study. Hypertension 62 (2): 331-336

5. Tanabe A, Naruse M, Naruse K et al (1997): Left ventricular hypertrophy is more prominent in patients with primary aldosteronism than in patients with other types of secondary hypertension. Hypertens Res 20 (2): 8590

6. Umemura S, Arima H, Arima S et al (2019): The Japanese Society of Hypertension Guidelines for the Management of Hypertension (JSH 2019). Hypertens Res 42 (9): 1235-1481

7. Beswick RA, Dorrance AM, Leite R \& Webb RC (2001): NADH/NADPH oxidase and enhanced superoxide production in the mineralocorticoid hypertensive rat. Hypertension 38 (5): 1107-1111

8. Ezekowitz JA \& McAlister FA (2009): Aldosterone blockade and left ventricular dysfunction: a systematic review of randomized clinical trials. Eur Heart J 30 (4): 469-477

9. Hu LJ, Chen YQ, Deng SB, Du JL \& She Q (2013): Additional use of an aldosterone antagonist in patients with mild to moderate chronic heart failure: a systematic review and meta-analysis. Br J Clin Pharmacol 75 (5): 1202-1212

10. Rubinshtein R, Kuvin JT, Soffler M et al (2010): Assessment of endothelial function by non-invasive peripheral arterial tonometry predicts late cardiovascular adverse events. Eur Heart J 31 (9): 1142-1148

11. Matsue Y, Yoshida K, Nagahori W et al (2014): Peripheral microvascular dysfunction predicts residual risk in coronary artery disease patients on statin therapy. Atherosclerosis 232 (1): 186-190

12. Torimoto K, Okada Y, Mori H \& Tanaka Y (2013): Relationship between fluctuations in glucose levels measured by continuous glucose monitoring and vascular endothelial dysfunction in type 2 diabetes mellitus. Cardiovasc Diabetol 12: 1

13. Torimoto K, Okada Y, Mori H \& Tanaka Y (2014): 
Low levels of 1,5-anhydro-D-glucitol are associated with vascular endothelial dysfunction in type 2 diabetes. Cardiovasc Diabetol 13: 99

14. Torimoto K, Okada Y, Mori H et al (2015): Effects of exenatide on postprandial vascular endothelial dysfunction in type 2 diabetes mellitus. Cardiovasc Diabetol 14: 25

15. Kurozumi A, Okada Y, Mori H et al (2016): Detrimental effects of high-fat diet loading on vascular endothelial function and therapeutic efficacy of ezetimibe and statins in patients with type 2 diabetes. Endocr J 63 (5): 431-440

16. Goshima Y, Okada Y, Torimoto K, Fujino Y \& Tanaka Y (2020): Changes in endothelial function during educational hospitalization and the contributor to improvement of endothelial function in type 2 diabetes mellitus. Sci Rep 10 (1): 15384

17. Tanaka K, Okada Y, Hajime M \& Tanaka Y (2021): Low Vitamin D Levels are Associated with Vascular Endothelial Dysfunction in Patients with Poorly Controlled Type 2 Diabetes: A Retrospective Study. J Atheroscler Thromb Jan 29. doi: 10.5551/jat.59113

18. Bonetti PO, Pumper GM, Higano ST, Holmes DR, Jr. , Kuvin JT \& Lerman A (2004): Noninvasive identification of patients with early coronary atherosclerosis by assessment of digital reactive hyperemia. J Am Coll Cardiol 44 (11): 2137-2141

19. Funder JW, Carey RM, Mantero F et al (2016): The Management of Primary Aldosteronism: Case Detection, Diagnosis, and Treatment: An Endocrine Society Clinical Practice Guideline. J Clin Endocrinol Metab 101 (5): 1889-1916

20. Tsuchiya K, Yoshimoto T \& Hirata Y (2009): Endothelial dysfunction is related to aldosterone excess and raised blood pressure. Endocr J 56 (4): 553-559

21. Nishizaka MK, Zaman MA, Green SA, Renfroe KY \& Calhoun DA (2004): Impaired endothelium-dependent flow-mediated vasodilation in hypertensive subjects with hyperaldosteronism. Circulation 109 (23): 28572861

22. Chou CH, Chen YH, Hung CS et al (2015): Aldosterone Impairs Vascular Smooth Muscle Function: From Clinical to Bench Research. J Clin Endocrinol Metab 100 (11): 4339-4347

23. Hao L, Kanno Y, Fukushima R, Watanabe Y, Ishida Y \& Suzuki H (2004): Effects of eplerenone on heart and kidney in two-kidney, one-clip rats. Am J Nephrol 24 (1): $54-60$

24. Kobayashi N, Hara K, Tojo A et al (2005): Eplerenone shows renoprotective effect by reducing LOX-1-mediated adhesion molecule, PKCepsilon-MAPK-p90RSK, and Rho-kinase pathway. Hypertension 45 (4): 538544

25. Sanz-Rosa D, Oubina MP, Cediel E et al (2005): Eplerenone reduces oxidative stress and enhances eNOS in SHR: vascular functional and structural consequences. Antioxid Redox Signal 7 (9-10): 1294-1301

26. Nishizaka MK, Zaman MA, Green SA, Renfroe KY \& Calhoun DA (2004): Impaired endothelium-dependent flow-mediated vasodilation in hypertensive subjects with hyperaldosteronism. Circulation 109 (23): 28572861

27. Macdonald JE, Kennedy N \& Struthers AD (2004): Effects of spironolactone on endothelial function, vascular angiotensin converting enzyme activity, and other prognostic markers in patients with mild heart failure already taking optimal treatment. Heart 90 (7): 765770

28. Hundemer GL, Curhan GC, Yozamp N, Wang M \& Vaidya A (2018): Cardiometabolic outcomes and mortality in medically treated primary aldosteronism: a retrospective cohort study. Lancet Diabetes Endocrinol 6 (1): 51-59

$$
\text { J UOEH } 43(4): 379-384(2021)
$$

